\title{
Studium der Vererbung des Geschlechts und des Wuchstyps beim Hanf*
}

WALTHER DIERKS † und REINHOLD VON SENGBUSCH

Max-Planck-Institut für Kulturpflanzenzüchtung, Hamburg-Volksdorf 


\section{Studies on the inheritance of sex and growth type in hemp'}

Summary. In dioecious hemp (variety 'Schurig-Markee') a sex ratio of 42 males: 58 females rather than the expected 1:1 ratio was found. From this we assumed sublethality of the males.

Progeny tests on the offspring from monoecious plants of female growth type and from those of male growth type with female plants of female growth type were carried out. They showed that monoecious plants of female growth type are of the XX genotype, those of male growth type XY.

Among the offspring of monoecious plants with male growth type the expected sex ratio was $3: 1$ or $2: 1$. The actual ratio found corresponded to dioecious segregation. We assumed lethality of $\mathrm{Y}$ eggcells to be responsible for the abnormal ratios in the offspring of monoecious plants with male growth type. When the latter were crossed with plants of female growth type only offspring with female growth type resulted. Reciprocal crosses, however, showed only the dioecious segregation ratios. Thus lethality of $\mathrm{Y}$ eggcells has been confirmed.

These results show why the breeding of monoecious hemp with male growth type has been an impossibility.

\section{Die beiden Wuchstypen des Hanfs}

Der Hanf ist eine ursprünglich rein diözische Pflanze mit einem starken Geschlechtsdimorphismus. Die seitenständigen Blütenstände der männlichen und weiblichen Pflanzen sind stark verschieden, und zwar sind die männlichen Blütenstände trugdoldig, sie bilden am oberen Teil der Stengel eine Art lockere Rispe. Die männlichen Blüten sind kurzgestielt. Der weibliche Blütenstand ist das laubige Sproßende. Die weiblichen Blüten sind ungestielt und stehen

* Herrn Prof. Dr. Hans Stubbe zum 65. Geburtstag gewidmet. zu zweit in den Achseln kleiner Laubblätter. Ein Unterschied $z$ wischen den weiblichen und männlichen Pflanzen besteht auch in der Lebensdauer. Während die Männchen nach dem Ausstäuben des Pollens absterben, benötigen die Weibchen noch $2-4$ Wochen bis zur Reife der Samen. Außerdem werden die Männchen etwa $20-25 \mathrm{~cm}$ länger als die Weibchen. Der Wuchstyp erweist sich den verschiedenen Außenbedingungen gegenüber als sehr stabil.

Neben den rein diözischen Formen gibt es aber auch monözische oder zwittrige Pflanzen, und zwar sowohl solche mit weiblichem als auch solche mit männlichem Habitus (Abb. 1-4).

\section{Vererbung des Wuchstyps}

Beim diözischen Hanf findet man auf 58 Pflanzen mit weiblichem Habitus etwa 42 Pflanzen mit männlichem Habitus, d. h. ein angenähertes 1:1-Verhältnis. Die Vererbung des Geschlechts geschieht, wie McPhee (1925) und Hrrata (1927) zeigen konnten, nach dem Homoheterogametie-Schema. Pflanzen mit weiblichem Habitus ergaben geselbstet oder untereinander bestäubt nur Weibchen. Sie sind also die XX-Typen. Nach Kreuzung mit männlichen Pflanzen traten dagegen Weibchen und Männchen auf, d. h. die Männchen repräsentieren den XY-Typ.

Wir nehmen mit GRISCH Ko (1937), aber im Gegensatz zu Horfmann $(1947$; 1952) an, daß die monözischen Formen mit weiblichem Habitus abgewandelte Weibchen, die monözischen Formen mit männlichem Habitus dagegen abgewandelte Männchen sind. Nach dieser Hypothese müßten die Monözisten mit weiblichem Wuchs XX-Typen und die Monözisten mit männlichem Wuchs XY-Typen sein. 


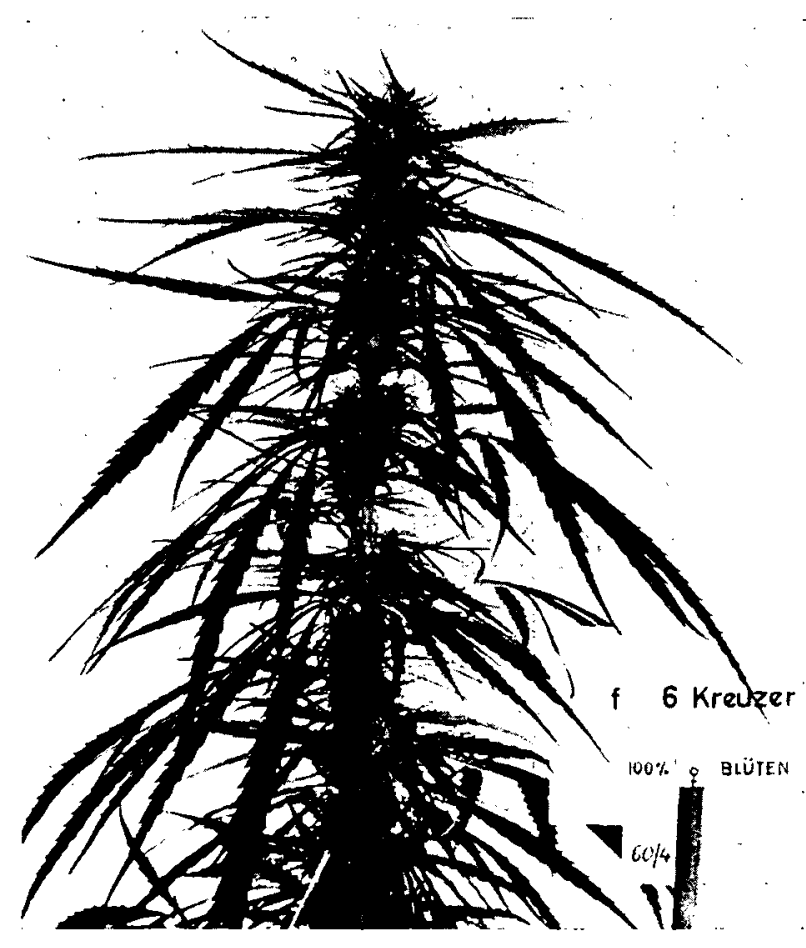

Abb. 1. Weibchen mit weiblichem Habitus.

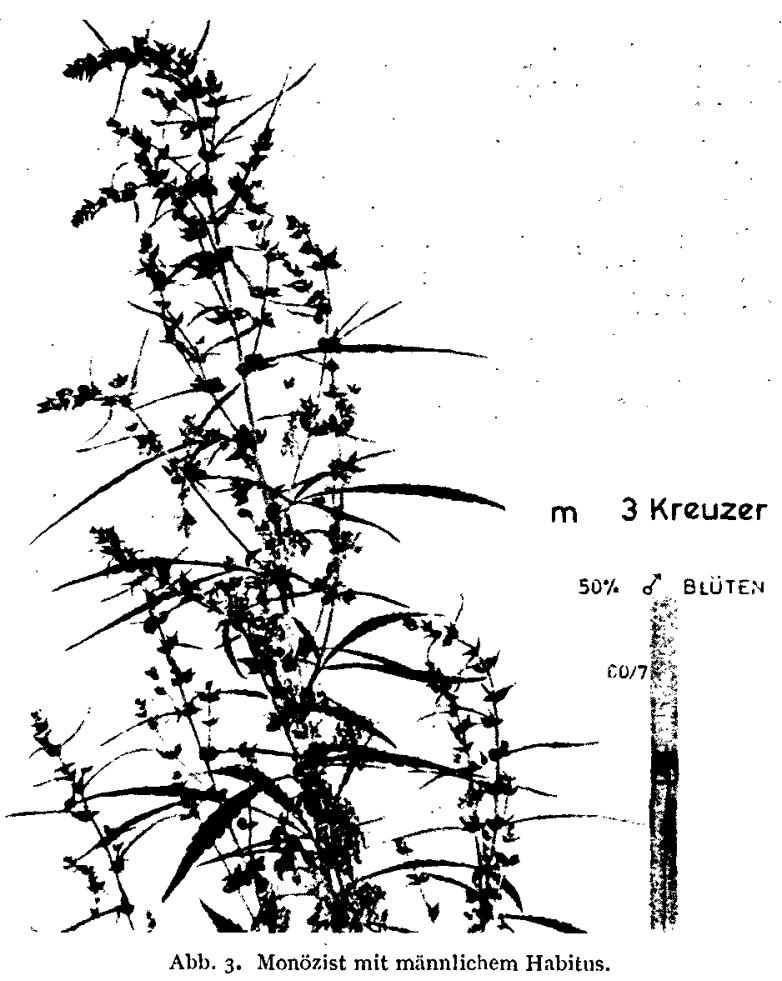

$F_{1}$ diözische Weibchen $\times$ Monözisten mit weiblichem Wuchs

Es ist dann $\mathrm{zu}$ erwarten, daß $\mathrm{F}_{1}-\mathrm{Nachkommen-}$ schaften der Kreuzung diözische Weibchen $\times$ Monözisten mit weiblichem Wuchs nur aus Pflanzen mit weiblichem Habitus bestehen. Eine 1952 angezogene derartige Nachkommenschaft von 24850 Pflanzen setzt sich folgendermaßen zusammen:

17860 Weibchen, 6990 Monözisten mit weiblichem Habitus, 139 normale Männchen $(0,56 \%)$.

Wenn man annimmt, daß diese Männchen aus Fremdbestäubung der weiblichen Mutterpflanze mit

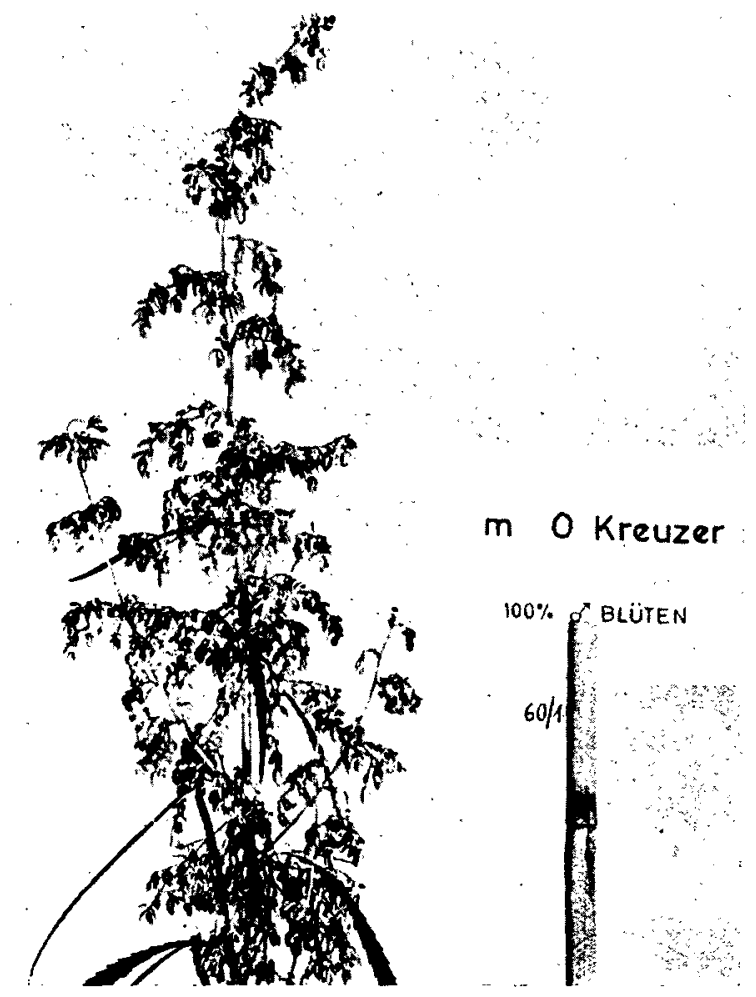

Abb. 2. Männchen mit männliciıem Habitus.

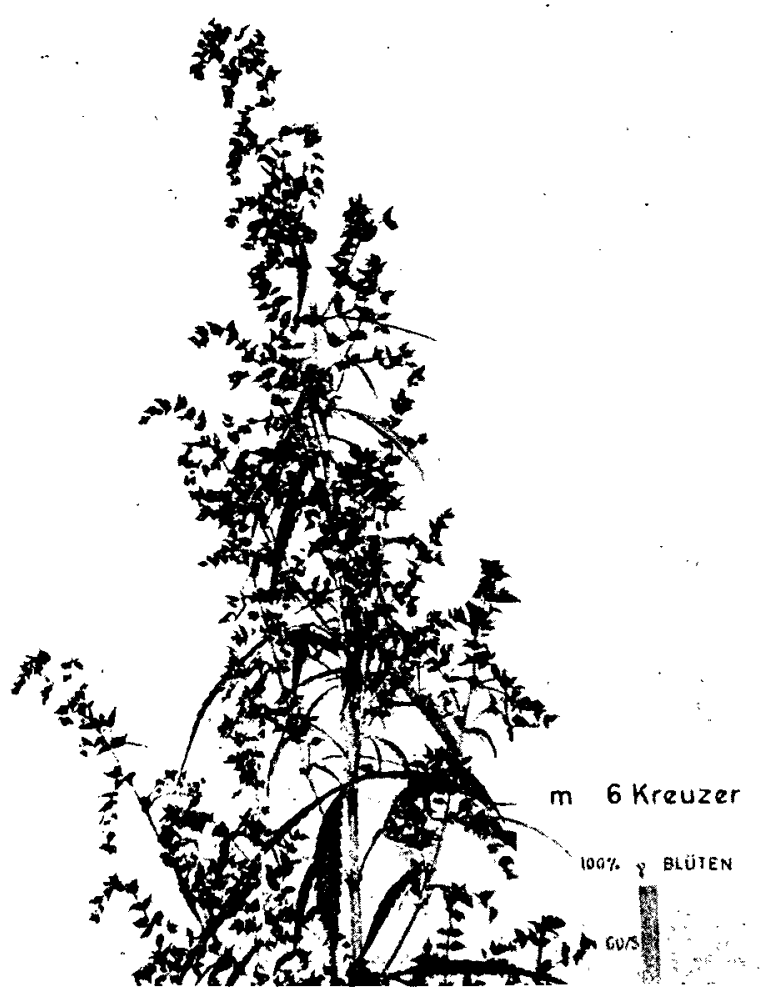

Abb. 4. Weibchen mit männlichem Habitus.

Pollen nicht früh genug entfernter diözischer Männchen entstanden sind, stimmt dieses Ergebnis mit der Hypothese überein.

$F_{1}$ Monözisten mit weiblichem Habitus $\times$ Monözisten mit weiblichem Habitus

Auch in dieser Nachkommenschaft dürften nach unserer Hypothese nur Pflanzen mit weiblichem 
Wuchs auftreten. In einem 1942 durchgeführten Versuch (NEUER u. v. SENGBUSCH, 1943) wurden unter 13003 Pflanzen 12886 Individuen mit weiblichem Habitus und 117 normale Männchen gefunden. Wiederum stimmt das Ergebnis mit der Hypothese überein, wenn man unterstellt, daß die Männchen durch unkontrollierte Fremdbestäubung entstanden sind.

Da HofFmanN $(1047 ; 1952)$ aber annimmt, da $B$ derartige Männchen in der $F_{1}$ herausspalten und nicht aus unbeabsichtigter Befruchtung durch diözische Männchen entstehen, ist geplant, diese beiden angeführten Kreuzungen noch einmal unter ganz streng kontrollierten Bedingungen zu wiederholen. Außerdem soll eine Kreuzung zwischen monözischen Pflanzen mit weiblichem Wuchs und diözischen Männchen durchgeführt werden. Die Nachkommenschaft muß, wenn unsere Hypothese richtig ist, je zur Hälfte aus Pflanzen mit weiblichem und Pflanzen mit männlichem Wuchs bestehen.

\section{$F_{1}$ diözische Weibchen $\times$ Monözisten mit männlichem Wuchs}

Wenn die Monözisten mit männlichem Habitus $\mathrm{XY}-\mathrm{Typen}$ sind, muB eine Kreuzung mit diözischen Weibchen entsprechend dem diözischen Spaltungsverhältnis Pflanzen mit männlichem und weiblichem Wuchs ergeben.

(Für den diözischen Schurig-Hanf wurde über 4 Jahre hin ein Verhältnis von 100 Männchen zu 136 Weibchen gefunden (Homogenität $P=0,79$ ). Dieses Ergebnis stimmt mit dem idealen 1:1-Verhältnis nicht überein $(P=0,002)$.)

In der Kreuzung diözische Weibchen (Fibridia) $\times$ Monözist mit männlichem Habitús kamen auf 46 Pflanzen mit männlichem Wuchs 62 mit weiblichem Habitus. Das entspricht dem diözischen Verhältnis (1 männlicher Habitus:1,36 weiblicher Habitus) sehr gut $(\mathrm{P}=0,90)$. Ebenso entsprach eine weitere Kreuzung (diözische Weibchen aus italienischem Hanf $\times$ Monözisten) mit einem Verhältnis von 111 Pflanzen mit männlichem Wuchs zu 120 Pflanzen mit weiblichem Wuchs den Erwartungen $(\mathrm{P}=0,065)$.

\section{$F_{1}$ Monözisten mit männlichem Habitus $\times$ Monözisten mit männlichem Habitus}

In dieser Kreuzung sind theoretisch verschiedene Aufspaltungen zu erwarten, je nach dem, ob die Y-Gameten subletal oder sogar letal sind oder nicht, und ob eventuelle YY-Zygoten lebensfähig sind oder nicht.

Die folgenden Schemata geben einen Uberblick über die jeweils zu erwartenden Aufspaltungen:

1. Y-Gameten voll funktionsfähig, YY-Zygoten lebensfähig

\begin{tabular}{c|c|c|c}
\hline Pollen: & $\mathrm{X}$ & $\mathrm{Y}$ & Aufspaltung \\
\hline Eizellen & & & Habitus männlich:weiblich \\
$\mathrm{X}$ & $1 \mathrm{XX}$ & $1 \mathrm{XY}$ & $=3(2 \mathrm{XY}, \mathbf{1} \mathrm{YY}): 1(1 \mathrm{XX})$ \\
$\mathrm{Y}$ & $1 \mathrm{XY}$ & $1 \mathrm{YY}$ &
\end{tabular}

1 a. Gameten subletal entsprcchend dem diözischen Aufspaltungsverhältnis, YY-Zygoten lebensfähig

\begin{tabular}{|c|c|c|c|}
\hline Pollen: & $1,36 \mathrm{X}$ & $1 \mathrm{Y}$ & Aufspaltung \\
\hline $\begin{array}{c}\text { Eizellen } \\
1,36 \mathrm{X} \\
1\end{array}$ & $\begin{array}{l}1,85 \times X \\
1,36 \times X\end{array}$ & $\mid \begin{array}{cc}1,36 X Y \\
1 & \mathrm{YY}\end{array}$ & $\begin{array}{l}\text { Habitus männlich: weiblich } \\
=3,7^{2: 1,85}=2: 1\end{array}$ \\
\hline
\end{tabular}

2. Y-Gameten voll funktionsfähig, YY-Zygoten letal

\begin{tabular}{c|c|c|l}
\hline Pollen: & $1 \mathrm{X}$ & $1 \mathrm{Y}$ & \multicolumn{1}{|c}{ Aufspaltung } \\
\hline Eizellen & & & Habitus männlich: weiblich \\
$1 \mathrm{X}$ & $1 \mathrm{XX}$ & $1 \mathrm{XY}$ & $=2: 1$ \\
$1 \mathrm{Y}$ & $1 \mathrm{XY}$ & - &
\end{tabular}

2a. Y-Gameten subletal, YY-Zygoten letal

\begin{tabular}{|c|c|c|c|}
\hline Pollen: & $1,36 \mathrm{X}$ & $1 Y$ & Aufspaltung \\
\hline $\begin{array}{l}\text { Eizellen } \\
1,36 \mathrm{X} \\
1\end{array}$ & $\begin{array}{l}1,85 \mathrm{XX} \\
1,36 \mathrm{XY}\end{array}$ & $\left|\begin{array}{c}1,36 \mathrm{XY} \\
-\end{array}\right|$ & $\begin{array}{l}\text { Habitus männlich: weiblich } \\
=2,72: 1,85=1,5: 1\end{array}$ \\
\hline
\end{tabular}

\begin{tabular}{c|c|c|l}
\hline Pollen: & $1 \mathrm{X}$ & $1 \mathrm{Y}$ & \multicolumn{1}{|c}{ Aufspaltung } \\
\hline $\begin{array}{c}\text { Eizellen } \\
1 \mathrm{X}\end{array}$ & $1 \mathrm{XX}$ & $1 \mathrm{XY}$ & $\begin{array}{l}\text { Habitus männlich: weiblich } \\
=1: 1\end{array}$
\end{tabular}

3a. Y-Eizellen letal, Y-Pollen subletal

\begin{tabular}{|c|c|c|c|}
\hline Pollen: & $1,36 \mathrm{X}$ & $1 Y$ & Aufspaltung \\
\hline $\begin{array}{c}\text { Eizellen } \\
1 \mathrm{X} \\
-\end{array}$ & $\begin{array}{c}1,36 \mathrm{XX} \\
-\end{array}$ & $1 Y Y$ & $\begin{array}{l}\text { Habitus männlich: weiblich } \\
=1: 1,36=0,74: 1\end{array}$ \\
\hline
\end{tabular}

Im Frühjahr und Sommer 1958 wurden je 14 bzw. 10 Nachkommenschaften von frei untereinander abgeblühten Monözisten mit männlichem Habitus untersucht. Folgende Aufspaltungen traten auf (m. H. bedeutet Pflanzen mit männlichem Habitus, w. H. bedeutet Pflanzen mit weiblichem Habitus) :

1. Frühjahr $195^{8}$ (Homogenität der zusammengefaßten 14 Nachkommenschaften $\mathrm{P}=0, \mathbf{1 7})$ :

Befund: $166 \mathrm{~m}$. H.: $212 \mathrm{w}, \mathrm{H}$.

Erwartung für $0,74 \mathrm{~m} . \mathrm{H} .: 1 \mathrm{w} . \mathrm{H}$. (Schema $3 \mathrm{a}$ )

$160 \mathrm{~m}$. H.:218 w. H. ; $P=0,56$

Erwartung für $1 \mathrm{~m} . \mathrm{H} .: 1 \mathrm{w}$. H. (Schema 3)

189 m. H.: 189 w. H.; $\mathrm{P}=0,019$

Erwartung für 1,5 m. H.: 1 w. H. (Schema 2a)

227 m. H.: 151 w. H.; $\mathrm{P}=<10^{-9}$

Erwartung für $3 \mathrm{~m}$. H.: 1 w. H. (Schema 1)

284 m. H.: 94 w. H.; $\mathrm{P}=<10^{-10}$

2. Sommer 1958 (Homogenität der zusammengefaßten 10 Nachkommenschaften $\mathrm{P}=0,54)$ :

Befund: $91 \mathrm{~m}$. H.: 128 w. H.

Erwartung für 0,74 m. H.: 1 w. H. (Schema 3a)

93 m. H.: $126 \mathrm{w}$. H.; $\mathrm{P}=0,78$

Erwartung für $1 \mathrm{~m} . \mathrm{H} .: 1 \mathrm{w} . \mathrm{H}$. (Schema 3)

109, 5 m. H.:109,5 w. H.; $\mathrm{P}=0,013$

Erwartung für 1,5 m. H.:1 w. H. (Schema 2a)

$131 \mathrm{~m}$. H.:88 w. H.; $\mathrm{P}=<10^{-7}$

Erwartung für $3 \mathrm{~m}$. H.: 1 w. H. (Schema 1)

164 m. H.: 55 w. H.; $\mathrm{P}=<10^{-10}$

Die Ergebnisse zeigen mit einem $\mathrm{P}$ von $0,56 \mathrm{bzw}$. 0,78 eine sehr gute Übereinstimmung der beobachteten Spaltungen mit dem Verhältnis $0,74 \mathrm{~m}$. H. zu $1 \mathrm{w} . \mathrm{H}$., d. h. mit der Aufspaltung, die zu erwarten ist, wenn die Y-Eizellen letal und die Y-Pollen subletal sind (Schema 3a). Dagegen ergibt sich eine hochsignifikante Abweichung von dem Spaltungsverhältnis $1,5 \mathrm{~m} . \mathrm{H} .: 1 \mathrm{w} . \mathrm{H}$., das zu erwarten ist, wenn entsprechend Schema 2 a die Y-Gameten vermindert lebensfähig und die YY-Zygoten nicht lebensfähig sein würden ( $\left.\mathrm{P}=10^{-9} \mathrm{bzw} \cdot 10^{-7}\right)$.

Noch höher signifikant ist dementsprechend die Abweichung der gefundenen Spaltungszahlen vom $3 \mathrm{~m}$. H.:1 w. H.-Verhältnis (Schema 1), das man 
erwarten müßte, wenn sowohl die männlichen und weiblichen $\mathrm{Y}$-Gameten als auch die YY-Zygoten lebensfähig wären $\left(\mathrm{P}<10^{-10}\right)$.

Um die Letalität der Y-Eizellen nachzuweisen, wurde im Winter $1958 / 59$ eine reziproke Kreuzung zwischen Monözisten mit männlichem Habitus und Monözisten mit weiblichem Habitus durchgeführt.

Werden Monözisten mit männlichem Wuchs als weiblicher Elter benutzt, muß bei Ausfall der Y-Eizellen die Nachkommenschaft rein weiblich sein, wie das folgende Schema zeigt:

\begin{tabular}{c|c|c}
\hline Pollen: & $\mathrm{X}$ & $\mathrm{X}$ \\
\hline Eizellen & $\mathrm{XX}$ & $\mathrm{XX}$ \\
$\mathrm{X}$ & - & -
\end{tabular}

Dienen dagegen die Monözisten mit weiblichem Habitus als Mutter, ist eine Aufspaltung entsprechend dem diözischen Verhältnis zu erwarten:

\begin{tabular}{c|c|c}
\hline Pollen: & $1,36 \mathrm{X}$ & $1 \mathrm{Y}$ \\
\hline Eizellen & & \\
$\mathrm{X}$ & $1,36 \mathrm{XX}$ & $1 \mathrm{XY}$ \\
$\mathrm{X}$ & $1,36 \mathrm{XX}$ & $1 \mathrm{XY}$
\end{tabular}

In der Kreuzungsrichtung Monözisten mit männlichem Habitus $\times$ Monözisten mit weiblichem Habitus wurden 20 Nachkommenschaften erhalten und geprüf. Von den 451 Pflanzen hatten 449 weiblichen Wuchs und 2 männlichen Wuchs. Das Ergebnis entspricht den Erwartungen, wenn man annimmt, $\mathrm{da} B$ die beiden Pflanzen mit männlichem Habitus durch versehentliche Selbstung entstanden sind.

Bei der großen Anzahl von Kastrationen, die in diesem Versuch ausgeführt werden mußten, war solch ein Fehler praktisch nicht $z u$ verhindern.

In der reziproken Kreuzungsrichtung, Monözisten mit weiblichem Habitus $\times$ Monözisten mit männlichem Habitus, wurde dagegen erwartungsgemä $\beta$ eine dem diözischen Verhältnis entsprechende Aufspaltung in männliche und weibliche Wuchstypen gefunden:

Befund: 314 männl. Habitus : 421 weibl. Habitus Erwartung: 311,4 männl. Habitus:423,6 weibl. Habitus

$$
\mathrm{P}=0,84
$$

Unsere Hypothese, daß die von uns untersuchten Pflanzen mit männlichem Wuchs nicht in der Lage sind, lebensfähige Y-Eizellen auszubilden, konnte also bestätigt werden.

Wir können damit gleichzeitig erklären, warum es bisher nicht gelungen ist, eine monözische Hanfsorte mit männlichem Wuchs konstant zu züchten.

Um dieses Ziel doch noch zu erreichen, müssen Pflanzen mit lebensfähigen Y-Eizellen ausgelesen werden. Weiterhin ist nötig, daß die in ihrer Nachkommenschaft auftretenden YY-Zygoten nicht letal sind. Solche YY-Pflanzen wären leicht daran zu erkennen, daB ihre Nachkommenschaft ausschließlich aus Pflanzen mit männlichem Habitus bestünde, gleichgültig ob sie von YY-, XY-oder XX-Typen bestäubt wurden.

\section{Zusammenfassung}

Beim diözischen Hanf (Sorte Schurig-Markee) wurde ein vom 1:1-Verhältnis abweichendes Geschlechtsverhältnis von 42 Männchen : 58 Weibchen gefunden. Danach war eine Subletalität der Männchen anzunehmen.

Es wurden Nachkommenschaftsprüfungen der Kreuzungen einerseits von Monözisten mit weiblichem Habitus und andererseits solchen mit männlichem Habitus mit Weibchen mit weiblichem Habitus durchgeführt. Sie ergaben die Zugehörigkeit der Monözisten mit weiblichem Habitus zum XX-Typ und der Monözisten mit männlichem Habitus zum XY-Typ.

Bei den Nachkommenschaften von Monözisten mit männlichem Habitus war ein Spaltungsverhältnis von $3: 1$ oder $2: 1 \mathrm{zu}$ erwarten. Gefunden wurde ein Verhältnis, das der diözischen Spaltung entspricht.

Für das andersartige Verhalten der Nachkommenschaften eines Monözisten mit männlichem Habitus mußte die Letalität der Y-Eizellen angenommen werden. Kreuzungen von Monözisten mit männlichem Habitus mit solchen mit weiblichem Habitus ergaben Nachkommenschaften mit rein weiblichem Habitus.

Die reziproke Kreuzung dagegen spaltete nach dem diözischen Verhältnis. Damit wurde die Letalität der Y-Eizelle bestätigt.

Dieses Ergebnis zeigt, weshalb ein monözischer Hanf mit männlichem Habitus nicht gezüchtet werden konnte.

Für ihre fleißige und sorgfältige Mitarbeit bei der Durchführung der Versuche sei Fräulein Helga Martens auch an dieser Stelle herzlich gedankt.

\section{Literatur}

1. GRischKo, N. N.: Odnowremenno sosrewajuschtschaja Konoplja, Nowoje w selskom chosaistwe, Wipusk 5 (1937). - 2. Hirata, K.: Sex determination in hemp (Cannabis sativa). J. Genet. 19, 65-79 (1927). 3. Hoffmann, W.: Gleichzeitig reifender Hanf. Der Züchter 13, 277-283 (1941). - 4. HoffmanN, W.: Die Vererbung der Geschlechtsformen des Hanfes (Cannabis sativa L.). I. Der Züchter 17/18, 257-277 (1947). 5. Hoffmann, W.: Die Vererbung der Geschlechtsformen des Hanfes. II. Der Züchter 22, 147-158 (1952). 6. 'KöHLER, D.: Geschlechtsbestimmung bei Blütenpflanzen. Ergebnisse der Biologie 27, 98-115 (1964). 7. McPheE, H.: The genetics of sex in hemp. Journ. Agric. Res. 31, 935 (1925). - 8. Neuer, H., u. R. v.SENGBUSCH: Die Geschlechtsvererbung bei Hanf und die Züchtung eines monözischen Hanfes. Der Züchter 15, 49-62 (1943). - 9. v. Sengbusch, R.: Die Erzeugung von Hanfstecklingen und die Umstimmung von der generativen in die vegetative Phase und umgekehrt. Vortrag gehalten am 18.2. 58 in Versailles; unveröffentlicht. 10. v. Sengbusch, R.: Beitrag zum Geschlechtsproblem bei Cannabis sativa. Z. Vererbungsl. 80, 616-618 (1942). - 11. v. Sengbusch, R.: Ein weiterer Beitrag zur Vererbung des Geschlechts bei Hanf als Grundlage für die Züchtung eines monözischen Hanfes. Z. Pflanzenzüichtung $31,319-338$ (1952). 